PROCEEDINGS OF THE

AMERICAN MATHEMATICAL SOCIETY

Volume 125, Number 11, November 1997, Pages 3293-3297

S 0002-9939(97)04162-2

\title{
A SYMMETRY PROBLEM FROM PROBABILITY
}

\author{
STEPHEN J. FROMM AND PATRICK MCDONALD
}

(Communicated by Jeffrey Rauch)

\begin{abstract}
We examine solutions of two related boundary value problems for smooth domains in Euclidean space which arise from variational problems in probability. We show that the existence of solutions to each problem implies that the domain is a sphere.
\end{abstract}

\section{INTRODUCTION}

Let $D$ be a connected open domain in $\mathbf{R}^{n}$ with a smooth boundary $S$, where $S$ is an oriented, compact, embedded hypersurface. In this note we prove the following result.

Theorem 1. Let $u$ be the solution of

$$
\begin{gathered}
\Delta u=-1 \text { on } D, \\
u=0 \text { on } S,
\end{gathered}
$$

and let $U$ be the solution of

$$
\begin{aligned}
\Delta U & =-u \text { on } D, \\
U & =0 \text { on } S .
\end{aligned}
$$

Suppose that

$$
\frac{\partial u}{\partial n} \frac{\partial U}{\partial n}=k
$$

where $k$ is a constant and $\partial / \partial n$ is the inward-pointing normal derivative along $S$. Then $S$ is a sphere and $u$ and $U$ are radial.

This symmetry result is closely related to the fundamental result of Serrin $[\mathrm{S}]$, and our proof follows Serrin's.

We were led to Theorem 1 by a study of variational problems for functionals defined on the space of embedded, compact, oriented, unparametrized hypersurfaces in $\mathbf{R}^{n}$ which bound a connected open domain [KM2]. We denote this space by $\mathcal{S} H\left(\mathbf{R}^{n}\right)$. The functionals considered were defined in terms of exit times for Brownian motion.

Received by the editors June 5, 1996.

1991 Mathematics Subject Classification. Primary 35J40, 60J65, 58G32.

Key words and phrases. Poisson problem, overdetermined boundary value problem.

(C) 1997 American Mathematical Society 
Specifically, given $S \in \mathcal{S} H\left(\mathbf{R}^{n}\right)$, let $D=D(S)$ be the domain bounded by $S$ and let $|D|$ be the volume of $D$. Let $\tau_{S}$ be the first exit time of Brownian motion from $D(S)$; that is,

$$
\tau_{S}=\tau_{S}(\omega)=\inf \left\{t \geq 0 ; W_{t}(\omega) \in S\right\}
$$

where $\omega$ belongs to a probability space and $W_{t}$ is $n$-dimensional Brownian motion. Define a functional $\mathcal{V}: \mathcal{S} H\left(\mathbf{R}^{n}\right) \longrightarrow \mathbf{R}$ by

$$
\mathcal{V}(S)=\frac{1}{|D|} \int_{D} E_{x}\left(\tau_{S}^{2}\right)-\left(E_{x}\left(\tau_{S}\right)\right)^{2} d x
$$

where $E_{x}\left(\tau_{S}\right)$ is the expected value of the random variable $\tau_{S}$, given that Brownian motion starts at $x$. The functional $\mathcal{V}$ denotes the average variance of the first exit time for Brownian motion for the domain bounded by $S$. For $v_{0}$ an arbitrary constant, define $\mathcal{S} H_{v_{0}}\left(\mathbf{R}^{n}\right)$ by

$$
\mathcal{S} H_{v_{0}}\left(\mathbf{R}^{n}\right)=\left\{S \in \mathcal{S} H\left(\mathbf{R}^{n}\right):|D(S)|=v_{0}\right\} .
$$

Restrict $\mathcal{V}$ to the subspace $\mathcal{S} H_{v_{0}}\left(\mathbf{R}^{n}\right)$. By computing the first variation of the functional under volume-preserving infinitesimal variations of the domain $D(S)$, it follows that at critical points of $\mathcal{V}$, the overdetermined boundary value problem in Theorem 1 must admit a solution [KM2]. Thus, by Theorem 1, a critical point of $\mathcal{V}$ (restricted to $\mathcal{S} H_{v_{0}}\left(\mathbf{R}^{n}\right)$ ) must be a sphere.

Fix $x_{0} \in \mathbf{R}^{n}$. If

$$
\mathcal{S} H_{v_{0}}\left(\mathbf{R}^{n}, x_{0}\right)=\left\{S \in \mathcal{S} H_{v_{0}}\left(\mathbf{R}^{n}\right): x_{0} \in D(S)\right\},
$$

a similar symmetry problem is associated with the functional $\mathcal{E}: \mathcal{S} H_{v_{0}}\left(\mathbf{R}^{n}, x_{0}\right) \longrightarrow$ $\mathbf{R}$ defined by

$$
\mathcal{E}(S)=E_{x_{0}}\left(\tau_{S}\right) .
$$

At critical points of $\mathcal{E}$, solutions of (1.1) satisfy

$$
\frac{\partial u}{\partial n} \frac{\partial G}{\partial n}\left(x_{0}, \cdot\right)=k
$$

where $G\left(x_{0}, \cdot\right)$ is the Green's function for the domain $D(S)$ with pole at $x_{0}$ and $k$ is a constant [KM1]. For this symmetry problem, we have

Theorem 2. If $u$ satisfies (1.1) and (1.4), then $S$ must be a sphere centered at $x_{0}$.

\section{Proof of Theorem 1}

We follow most of the notation of Serrin's result in [S]; for the reader's convenience we recap the relevant parts of the proof there. Fix a hyperplane $T_{0} \subset \mathbf{R}^{n}$ not intersecting $D$. Move the hyperplane parallel to itself, toward $D$; it will eventually cut off from $D$ an open cap, $\Sigma(T)$, where $T$ is parallel to $T_{0}$ and intersects $D$. The reflection of $\Sigma(T)$ in $T, \Sigma^{\prime}(T)$, will initially lie in $D$. This will remain true as $T$ continues to move into $D$, until one of the following two conditions occurs:

(i) $\Sigma^{\prime}(T)$ is internally tangent to $S$ at a point $P$ not on $T$, or

(ii) $T$ is orthogonal to $S$ at some point $Q$.

Below, we will assume $T$ satisfies either (i) or (ii) above. Define a function $V$ in $\Sigma^{\prime}=\Sigma^{\prime}(T)$ by $V(x)=U\left(x^{\prime}\right)$, where $x^{\prime}$ is the reflection of $x$ in $T$. Let $v(x)=u\left(x^{\prime}\right)$ for $x \in \Sigma^{\prime}$; note that $\Delta V(x)=-v(x)$ and $\Delta v=-1$.

Moreover, $V$ and $v$ satisfy the following boundary conditions in $\Sigma^{\prime}$ : on $T \cap \partial \Sigma^{\prime}$, $V=U$ and $v=u$; on the remainder, $T^{c} \cap \partial \Sigma^{\prime}, V=0$ and $v=0$. Also, on this latter 
set, $U \geq 0$ and $u \geq 0$. This follows because $\Sigma^{\prime} \subset D$ and the maximum principle for the laplacian shows that $U$ and $u$ are positive in $D$.

As pointed out in [S], we will have shown $S$ to be a sphere if, for arbitrary initial $T_{0}, D$ is symmetric about the hyperplane $T$ satisfying (i) or (ii) above. The symmetry of $D$ about $T$ follows if either $U \equiv V$ or $u \equiv v$ in $\Sigma^{\prime}$. We assume neither condition holds and obtain a contradiction.

If $u \not \equiv v$ in $\Sigma^{\prime}$, then by the maximum principle applied to $u-v$ it follows that $u-v>0$ in the interior of $\Sigma^{\prime}$. Since $\Delta(U-V)=v-u<0$ in $\Sigma^{\prime}$, it similarly follows that $U>V$ in $\Sigma^{\prime}$.

First suppose $T$ satisfies condition (i) above. As in [S], the boundary point maximum principle shows that

$$
\frac{\partial}{\partial n}(u-v)>0 \text { and } \frac{\partial}{\partial n}(U-V)>0 \text { at } P .
$$

This contradicts the fact that at $P$,

$$
\frac{\partial u}{\partial n} \frac{\partial U}{\partial n}=k \text { and } \frac{\partial v}{\partial n} \frac{\partial V}{\partial n}=k .
$$

Second, suppose $T$ satisfies condition (ii) above. Choose coordinates in $\mathbf{R}^{n}$ so that the origin is at $Q$, the positive $x_{n}$-axis has the same direction as the inward normal to $S$ at $Q$, and the positive $x_{1}$-axis is normal to $T$, pointing away from $\Sigma^{\prime}$.

Let $\vec{s}$ be a vector at $Q$ which is nontangential with respect to $\partial \Sigma^{\prime}$, and let $\partial / \partial s$ be the corresponding directional derivative. We need the following result, essentially Lemma 1 of [S]:

Lemma. Suppose $w \in C^{2}\left(\overline{\Sigma^{\prime}}\right), \Delta w \leq 0$ and $w \geq 0$ in $\Sigma^{\prime}$, and $w=0$ at $Q$. Then if $w \not \equiv 0$, either

$$
\frac{\partial w}{\partial s}>0 \text { or } \frac{\partial^{2} w}{\partial s^{2}}>0 \text { at } Q
$$

We apply the lemma to both $w=u-v$ and $w=U-V$, with $\partial / \partial s=-\partial / \partial x_{1}+$ $\partial / \partial x_{n}$. Near $Q$,

$$
v\left(x_{1}, x_{2}, \ldots, x_{n}\right)=u\left(-x_{1}, x_{2}, \ldots, x_{n}\right) .
$$

Denoting $\partial / \partial x_{i}$ with a subscript $i$, it follows that $v_{n}=u_{n}$ at $Q$. Because $u=0$ on $S$, it follows easily that $\partial u / \partial x_{i}=0$ at $Q$ for $1 \leq i \leq n-1$. Hence $u_{1}=0$ and $v_{1}=0$ at $Q$, and thus

$$
\frac{\partial u}{\partial s}=\frac{\partial v}{\partial s}
$$

at $Q$. Similarly, $\partial U / \partial s=\partial V / \partial s$ at $Q$. By the Lemma and the assumptions that $U \not \equiv V$ and $u \not \equiv v$, it follows that

$$
\frac{\partial^{2}}{\partial s^{2}}(u-v)>0 \text { and } \frac{\partial^{2}}{\partial s^{2}}(U-V)>0 \text { at } Q .
$$

Now consider

$$
\frac{\partial^{2}}{\partial s^{2}}=\frac{\partial^{2}}{\partial x_{1}^{2}}+\frac{\partial^{2}}{\partial x_{n}^{2}}-2 \frac{\partial^{2}}{\partial x_{1} \partial x_{n}} .
$$

By (2.1), it follows that

$$
v_{11}=u_{11} \text { and } v_{n n}=u_{n n}
$$


Similar reasoning shows that

$$
V_{11}=U_{11} \text { and } V_{n n}=U_{n n} .
$$

Since $U$ and $u$ vanish on $S$, we may rewrite (1.3) as $\nabla U \cdot \nabla u=k$ on $S$, i.e.,

$$
\sum_{i=1}^{n} U_{i} u_{i}=k \text { on } S
$$

Differentiating this equality at $Q \in S$ in the direction $\partial / \partial x_{1}$ tangent to $S$ at $Q$, it follows that

$$
U_{n} u_{n 1}+u_{n} U_{n 1}=0
$$

because $U_{i}=0$ and $u_{i}=0$ at $Q$ for $1 \leq i \leq n-1$. Similarly, $V_{n} v_{n 1}+v_{n} V_{n 1}=0$ at $Q$. Because $U_{n}=V_{n}$ and $u_{n}=v_{n}$ at $Q$, we may subtract to obtain

$$
U_{n}\left(v_{n 1}-u_{n 1}\right)+u_{n}\left(V_{n 1}-U_{n 1}\right)=0 .
$$

Combining (2.3), (2.4), and (2.5), we find that

$$
U_{n} \frac{\partial^{2}}{\partial s^{2}}(u-v)+u_{n} \frac{\partial^{2}}{\partial s^{2}}(U-V)=0 \text { at } Q .
$$

But this contradicts (2.2), because both $U_{n}$ and $u_{n}$ are positive at $Q$.

Remark. Notice that $\Delta^{2} U=1$. Bennett [B] proves the following symmetry result for this fourth-order equation: if $U$ and its normal derivative vanish on $S$, and $\Delta U$ is constant on $S$, then $D$ is a ball. His proof follows the method of [W], rather than that of $[\mathrm{S}]$. The authors, however, were unable to prove the results here by that method.

\section{Proof of Theorem 2}

For convenience, write $G(x)=G\left(x_{0}, x\right)$ and choose the sign of $G$ so that $G>0$ in $D$. With that choice, $G(x)$ is determined by

$$
\begin{aligned}
\Delta G(x) & =-\delta_{x_{0}}(x) \text { in } D, \\
G & =0 \text { on } S
\end{aligned}
$$

where $\delta_{x_{0}}$ is the delta mass at $x_{0}$.

As above, the method of moving planes yields a $T$ satisfying (i) or (ii). Again, if $x^{\prime}$ is the reflection of $x$ in $T$, define $v(x)=u\left(x^{\prime}\right)$ for $x \in \Sigma^{\prime}=\Sigma^{\prime}(T)$. Also define $\tilde{G}(x)=G\left(x^{\prime}\right)$. To prove Theorem 2 , it suffices to show that $D$ must be symmetric about $T$, as in the proof of Theorem 1 , and in addition that $x_{0} \in T$.

To begin with, assume $x_{0}$ indeed lies on $T$. The function $G-\tilde{G}$ is harmonic in $\Sigma^{\prime}$, vanishing on $T \cap \partial \Sigma^{\prime}$ and nonnegative on $T^{c} \cap \partial \Sigma^{\prime}$. The proof of Theorem 1 , with $G-\tilde{G}$ replacing $U-V$, shows that $v \equiv u$ and $\tilde{G} \equiv G$ in $\Sigma^{\prime}$. Hence $D$ is symmetric about $T$.

We next show that $x_{0} \notin T$ inevitably leads to a contradiction.

Case one: $x_{0} \in \Sigma^{\prime}$. Because $\Delta(G-\tilde{G})=-\delta_{x_{0}}, G-\tilde{G}$ is still superharmonic in $\Sigma^{\prime}$, hence $G-\tilde{G}>0$ there. Following the techniques of Theorem 1, we are led to a contradiction. 
Case two: $x_{0} \notin \Sigma^{\prime} \cup T \cup \Sigma$. Because $G-\tilde{G}$ is harmonic in $\Sigma^{\prime}$ in this case, $G \geq \tilde{G}$ by the maximum principle. (If $x_{0} \in \partial \Sigma^{\prime}$, the maximum principle may still be used, since $\tilde{G}(x) \rightarrow 0$ and $G(x) \rightarrow+\infty$ as $x \rightarrow x_{0}$.) By the methods of Theorem 1, it follows that $D$ is symmetric about $T$, and $D=\Sigma^{\prime} \cup(T \cap D) \cup \Sigma$. But this contradicts $x_{0} \in D$ and $x_{0} \notin \Sigma^{\prime} \cup T \cup \Sigma$.

Case three: $x_{0} \in \Sigma$. In $\Sigma^{\prime}, \Delta(G-\tilde{G})=\delta_{x_{0^{\prime}}}$, so $G-\tilde{G}$ is now subharmonic, preventing direct treatment of $u-v$ and $G-\tilde{G}$. A contradiction is obtained by considering the family $\left\{T_{1}\right\}$ of moving planes which are parallel to $T$ but which start at an initial position on the opposite side of $D$ and move in the direction opposite to that of the family which led to $T$. Again, it is required that the reflected cap $\Sigma^{\prime}\left(T_{1}\right)$ remain in $D$. The family $\left\{T_{1}\right\}$ will reach a plane $T_{1}^{*}$ satisfying (i) or (ii) above. In doing so, however, $\left\{T_{1}\right\}$ will never move past $T$ because of the conditions $\Sigma^{\prime}\left(T_{1}\right) \subset D$ and $\Sigma^{\prime}(T) \subset D$. Since $x_{0} \in \Sigma \equiv \Sigma(T)$, it follows that $x_{0} \notin \Sigma\left(T_{1}^{*}\right)$ and $x_{0} \notin T_{1}^{*}$, so that either case one or case two above holds for $T_{1}^{*}$ replacing $T$. Both those cases, however, resulted in contradictions.

\section{REFERENCES}

[B] A. Bennett, Symmetry in an overdetermined fourth order elliptic boundary value problem, SIAM J. Math. Anal. 17 (1986), 1354-1358. MR 87i:35059

[KM1] K. K. J. Kinateder and P. McDonald, Brownian functionals on hypersurfaces in Euclidean space, Proc. Amer. Math. Soc. 125 (1997), 1815-1822. CMP 97:07

[KM2] K. K. J. Kinateder and P. McDonald, Hypersurfaces in $\mathbf{R}^{d}$ and the variance of exit times for Brownian motion, Proc. Amer. Math. Soc. (to appear). CMP 96:16

[S] J. Serrin, A symmetry problem in potential theory, Arch. Rational Mech. Anal. 43 (1971), 304-318. MR 48:11545

[W] H. F. Weinberger, Remark on the preceding paper of Serrin, Arch. Rational Mech. Anal. 43 (1971), 319-320. MR 48:11546

Department of Mathematics, University of Wyoming, Laramie, Wyoming 82071

E-mail address: fromm@uwyo.edu

Department of Mathematics, The Ohio State University, Columbus, Ohio 43210

Current address: Department of Mathematics, New College, University of South Florida, Sarasota, Florida 34243

E-mail address: pmacdona@virtu.sar.usf.edu 